\title{
Productividad de tres híbridos experimentales de maíz amarillo duro (Zea mays L.) bajo condiciones climáticas de la costa norte del Perú
}

\section{Productivity of three experimental hybrids of hard yellow corn (Zea mays L.) under climatic conditions of the north coast of Peru}

\author{
Isaac Cieza-Ruiz ${ }^{1, a, *}$, Tito R. Vásquez-Rojas²,b
}

\footnotetext{
${ }^{1}$ Estación Experimental Agraria Vista Florida, Instituo Nacional de InnovaciónAgraria (INIA), Lambayeque, Perú.

${ }^{2}$ Universidad Autónoma Chapingo, Chapingo, México.

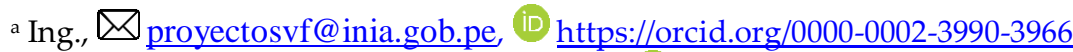

b Ph.D., 囚 troquevasquezrojas@ gmail.com, (iD https://orcid.org/0000-0003-1947-0363

* Autor de Correspondencia: Tel. +51978781863

http://dx.doi.org/10.25127/riagrop.20221.781
}

http://revistas.untrm.edu.pe/index.php/RIAGROP revista.riagrop@untrm.edu.pe

Recepción: 10 de noviembre 2021

Aprobación: 12 de diciembre 2021

Este trabajo tiene licencia de Creative Commons. Attribution-NonCommercial-ShareAlike $\quad 4.0$ International Public License - CC-BY-NC-SA 4.0

\section{Resumen}

El cultivo de maíz representa un alimento importante, tanto para humanos como animales. Actualmente, la producción de maíz no cubre la demanda nacional. El objetivo del trabajo fue evaluar la productividad de tres híbridos promisorios y dos híbridos comerciales en la EEA Vista Florida - Picsi. Para ello, se evaluaron cinco tratamientos: tres híbridos simples y dos híbridos comerciales, mediante el diseño en bloques completamente al azar con cuatro repeticiones. La densidad de siembra utilizada fue de 66,6666 plantas por hectárea. La dosis de N-P2O5-K2O fue de 240-140-160 $\mathrm{kg} / \mathrm{ha}$. Se evaluaron variables agronómicas como días a la floración masculina y femenina, altura de planta, altura a la mazorca, diámetro de tallo, longitud y diámetro de mazorca, hileras de mazorca y peso de mazorca. Los resultados encontrados mostraron los mayores rendimientos expresados por los híbridos promisorios CL02450 x CML287 y CLRYN017 x CL02450 con $9704.8 \mathrm{Kg}$ ha-1 y $8938.6 \mathrm{Kg}$ ha-1, respectivamente. Los híbridos AGRHICOL XB-8010 evidenciaron ser los más precoces de 
todos. La mayor altura y peso de mazorca se registró para INIA 619 Megahíbrido, que representa buena adaptabilidad a la costa norte del Perú, en relación a rendimiento de grano.

Palabras claves: Adaptación, rendimiento, promisorio, precocidad, Megahíbrido.

\begin{abstract}
The cultivation of corn represents an important food for both humans and animals. Currently, the production of corn does not cover the national demand. The objective of the work was to evaluate the productivity of three promising hybrids and two commercial hybrids in the EEA Vista Florida - Picsi. Is evaluated five treatments: three simple hybrids and two commercial hybrids, using the completely randomized block design with four replications. The sowing density used was 66.6666 plants per hectare. The dose of N-P2O5-K2O was 240-140-160 kg / ha. Agronomic variables such as male and female days to flowering, plant height, ear height, stem diameter, ear length and diameter, ear rows and ear weight were evaluated. The results found showed the highest yields expressed by the promising hybrids CL02450 x CML287 and CLRYN017 x CL02450 with 9 $704.8 \mathrm{Kg}$ ha-1 and $8938.6 \mathrm{Kg}$ ha-1, respectively. The AGRHICOL XB-8010 hybrids showed to be the earliest of all. The highest height and weight of the ear was registered for INIA 619 Megahybrid, which represents good adaptability to the north coast of Peru in relation to grain yield.
\end{abstract}

Keywords: Adaptation, performance, promising, precocity, Megahybrid.

\section{INTRODUCCIÓN}

La producción de maíz amarillo duro en el Perú es de 1262279 toneladas (MINAGRI, 2018). El maíz amarillo duro es uno de los principales alimentos para la industria peruana avícola y ganadera. Tiene la ventaja de ser cosechado casi todo el año, de preferencia en la costa peruana. Sin embargo, su producción nacional de 1.1 millones de toneladas no es del todo suficiente, por lo que resulta necesario importar de otros países (Caretas, 2021). Ante esto, en el 2021, se importó 1381002 toneladas por un valor de US\$ 383 millones. Estas cifras evidenciaron un incremento de 6,05\%, en cuanto a volumen, y 49,63\%, en el 2021 (La cámara, 2021). A nivel nacional, es notable una baja productividad del maíz amarillo duro con un rendimiento promedio nacional de $4.7 \mathrm{t} / \mathrm{ha}$ (Hortus, 2020). El rendimiento promedio del principal país productor, Estados Unidos, es 10.96 t/ha (MIDAGRI, 2021).

El desarrollo de híbridos de maíz involucra líneas endogámicas, progenitores no endogámicos o una combinación de líneas y progenitores no endogámicos. El número de progenitores involucrados en la formación de híbridos puede variar desde un mínimo de dos progenitores a un máximo de cuatro. Los híbridos se pueden agrupar en dos grandes clases: híbridos convencionales y no convencionales (Vasal et al., 1988)

Cuando las pruebas de comportamiento de variedades se realizan convencionalmente, ofrecen información sobre la interacción genotipo ambiente, que no es un indicativo de la estabilidad de las variedades evaluadas (Córdova, 1978). Por eso, el análisis de estabilidad es un instrumento en la identificación de germoplasma de gran 
potencial para los programas de mejoramiento. En base a la interpretación de los parámetros de estabilidad, Carballo y Márquez (1970) clasifican a una variedad de "estable" cuando Bi = 1 y S2 di=0. Los híbridos, desarrollados por el INIA, representan una alternativa frente a efectos climáticos adversos, sucedidos en las últimas campañas agrícolas por efecto del fenómeno El Niño y El Niño Costero, con temperaturas superiores a $\operatorname{los} 30{ }^{\circ} \mathrm{C}$, que provoca una tropicalización de la costa norte $\mathrm{y}$ perjudica los niveles de productividad del cultivo de maíz.

En este estudio, se evalúo la respuesta productiva de tres híbridos promisorios seleccionados por su potencial productivo en los años 2015 y 2016, como una alternativa para el valle agrícola de Chancay - Lambayeque y otros valles maiceros en el Perú.

\section{MATERIALES Y MÉTODOS}

El estudio se realizó en el valle ChancayLambayeque, distrito de Picsi, en campos de la Estación Experimental Agraria Vista Florida, con coordenadas, 06² $43^{\prime} 42^{\prime \prime}$ latitud sur y 794' $50^{\prime \prime}$ longitud oeste a $42 \mathrm{~m} \mathrm{s.} \mathrm{n.} \mathrm{m.} \mathrm{(figura}$ 1). La temperatura más alta se presentó en el mes de marzo $\left(33^{\circ} \mathrm{C}\right)$ y la mínima en el mes de agosto con $14.4{ }^{\circ} \mathrm{C}$. La mayor radiación se presentó en el mes de abril, con 38835 Joules m 2; y la mínima radiación en el mes de julio con 22553 Joules $\mathrm{m}^{-2}$. Estas condiciones no fueron muy favorables para el desarrollo de las plantas que tuvieron altas temperaturas y baja radiación en la fase reproductiva.

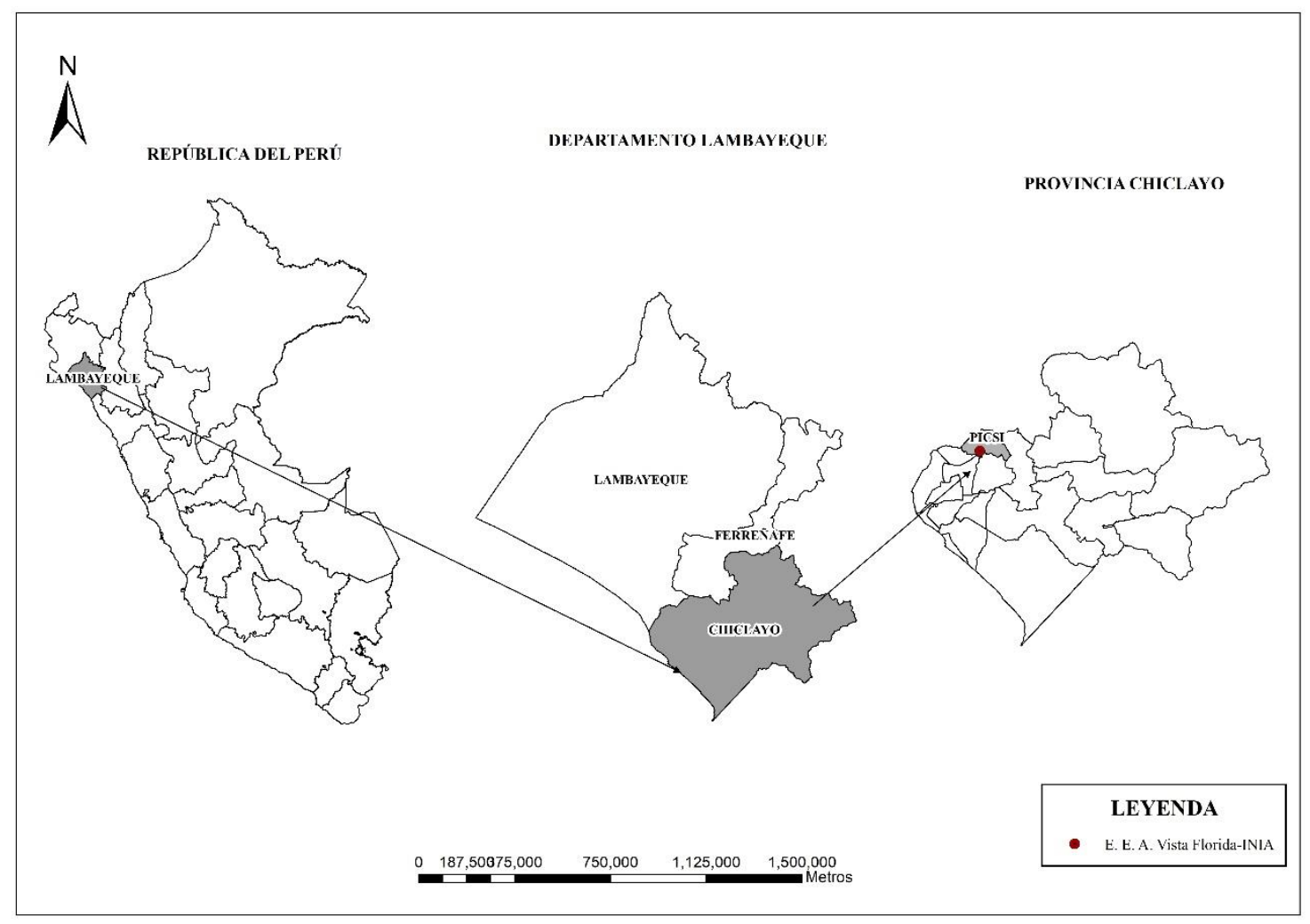

Figura 1. Lugar de ejecución del experimento. 
El suelo del área experimental fue de origen aluvial, de textura franca, con contenido de materia orgánica baja (2\%), fósforo medio (7.6 ppm), disponibilidad alta de potasio (327 ppm). El pH fue neutro (7).

Se estabecieron cinco tratamientos de estudio. De ellos, tres fueron híbridos simples promisorios y dos híbridos comerciales. La unidad experimental fue una parcela de $60 \mathrm{~m}^{2} \mathrm{y}$ con riego por goteo y un distanciamiento de 0.75 $\mathrm{m}$ entre cintas y 0.4 entre golpes.

El diseño empleado fue el de bloques completos al azar (DBCA), con cuatro repeticiones. Los híbridos promisorios en estudio fueron: CL02450 x CML287 (T1), CLRYNO17xCL02450 (T2), CML161xCL02410 (T3), y dos híbridos comerciales como INIA 619 - Megahíbrido (T4), AGRHICOL XB-8010 (T5).

La siembra se realizó el 28 de marzo del 2017. La densidad de siembra de los híbridos fue de 66,666 plantas ha-1, con la dosis de fertilización de 240-140-160 kg ha-1 de N-P2O5-K2O, el nitrógeno, a base de urea y sulfato de amonio, el fósforo como fosfato monoamónico y el potasio a base de sulfato de potasio.

La mezcla de fertilizantes se aplicó en estado vegetativo V4 (4 hojas), con la dosis 60-140-80 kg ha $^{-1}$. El $\mathrm{N}$ como sulfato de amonio y urea, la segunda dosis de 120 y $80 \mathrm{Kg} \mathrm{ha}^{-1}$ de $\mathrm{N}$ (urea) y K2O a la sexta hoja (V6) y la tercera dosis de N (urea) a la décina hoja (V10), con 60 kg ha-1.

El control de malezas se realizó con herbicida sistémico no selectivo (glifosato), antes de la siembra. La plaga insectil más importante fue el gusano cogollero (Spodoptera frugiperda), que fue controlado con aplicaciones de Thiodicarb al 30 $\%$ de concentración.
La unidad experimental estuvo conformada por 10 surcos, de 8 metros de largo por 7.5 metros de ancho. La distancia entre surco fue de $0.75 \mathrm{~m}$ por $0.4 \mathrm{~m}$ entre plantas, con un total de 21 golpes por surco, con dos plantas por golpe.

La cosecha se realizó en forma manual y se trabajaron los seis surcos centrales de la unidad experimental. Los rendimientos se ajustaron al $13 \%$ de humedad, mediante el cálculo con la siguiente fórmula:

Rendimiento de grano $=($ P.C. $x \% \mathrm{MS} \times \mathrm{\% G}) \mathrm{x}$ F.C.) / 8700

Donde PC es peso $(\mathrm{kg})$ de campo del total de mazorcas cosechadas en la parcela, \% MS es el porcentaje de materia seca, calculado con base en la muestra de grano de cinco mazorcas recién cosechadas, \% G represeta el porcentaje de grano obtenido como el cociente entre el peso de grano y el de mazorca, FC es el factor de conversión a rendimiento por ha, que se obtiene dividiendo $10000 \mathrm{~m}^{2}$ entre el tamaño de la parcela útil determinado en $\mathrm{m}^{2}\left(36 \mathrm{~m}^{2}\right)$ y 8700 es una constante empleada para estimar el rendimiento con una humedad del grano del 13 $\%$.

También, se ejecutó evaluaciones de altura de planta (AP), altura de inserción de mazorca (AM), largo de mazorca (LM), diámetro de mazorca (DM), hileras de la mazorca (HM), peso de mazorca (PM), floración masculina (FM) cuando el 50\% de las plantas liberaron polen y floración femenina (FF) cuando el 50\% de las plantas expusieron los estigmas en al menos tres $\mathrm{cm}$.

Además, se realizó pruebas de varianzas homogéneas, mediante Bartlett, y prueba de normalidad de datos con Kolmogorov-Smirnov, 
para la comporbación de la normalidad de datos. Finalmente, se aplicó una prueba de comparación de medias de Tukey al $5 \%$ de error, con el paquete estadístico SAS.

\section{RESULTADOS Y DISCUSIÓN}

El análisis de varianza para rendimiento en grano mostró diferencias significativas entre los tratamientos (tabla 1). Según la prueba de significación de Tukey al 0.05 (tabla 2), los híbridos promisorios superan a los híbridos comerciales de maíz amarillo duro (tabla 1). Estas diferencias se pueden atribuir al número de hileras y peso de mazorca, tal como lo mencionan Chura y Tejada (2014).

Según los datos de tabla 2, los híbridos promisorios rindieron en promedio $21 \%$ más que los híbridos comerciales de maíz amarillo duro, $9704.8 \mathrm{~kg}$ ha-1 vs 7664.5 ha-1 del híbrido simple comercial más sobresaliente. El híbrido promisorio CL02450 x CML287, con 9704.8 $\mathrm{kg} /$ ha de grano, tiene productividad superior a los otros cuatro tratamientos $\mathrm{y}$, a su vez, tiene rendimiento similar al híbrido promisorio CLRYN017xCL02450 que rindió $8938.6 \mathrm{~kg} / \mathrm{ha}$. Estos resultados fueron similares a los reportados por Banda (2019), quien encontró $8629.87 \mathrm{~kg} / \mathrm{ha}$ para INIA 619-Megahíbrido y AGRHICOL XB-8010, que se ubicó en penúltimo lugar, con 7718.34, en similares condiciones.

En las variables de floración masculina y femenina, altura de planta, altura de inserción de mazorca, diámetro de tallo, largo de mazorca, hileras de mazorca y peso de mazorca, se detectaron diferencias altamente significativas entre híbridos (tabla 3), que puede significar respuestas aceptables porque responden positivamente en los diferentes ambientes donde se cultivaron (Gordon et al., 2006).

Tabla 1. Análisis de variancia para rendimiento en grano de cinco híbridos de maíz amarillo duro, al 0.05 de significancia. Picsi, Lambayeque, Perú. Verano, 2017

\begin{tabular}{lrrrrc}
\hline \multicolumn{1}{c}{ FV } & GL & \multicolumn{1}{c}{ S.C } & C.M & F cal Pr $>$ F & $\begin{array}{c}\text { Nivel de } \\
\text { significación }\end{array}$ \\
\hline Bloques & 3 & 1030528.73 & 343509.58 & 0.970 .4368 & N.S. \\
Tratamiento & 4 & 19908096.59 & 4977024.15 & 14.120 .0002 & $* *$ \\
Error & 12 & 4228805.69 & 352400.47 & & \\
Total & 19 & 25167431.01 & & & CV $=7.21 \%$ \\
\hline
\end{tabular}

Tabla 2. Prueba de Tukey al 0.05 para Rendimiento en grano de cinco híbridos de maíz amarillo duro. Picsi, Lambayeque, Perú. Verano, 2017

\begin{tabular}{lc}
\hline Tratamientos & Rendimiento kg ha-1 $\left.^{-*}\right)$ \\
\hline CL02450 x CML287 & $9704.8 \mathrm{a}$ \\
CLRYNO17 x CL02450 & $8938.6 \mathrm{ab}$ \\
CML161 x CL02410 & $8020.1 \mathrm{bc}$ \\
INIA 619 - Megahíbrido & $7664.5 \mathrm{bc}$ \\
AGRHICOL XB-8010 & $6838.6 \mathrm{c}$ \\
\hline
\end{tabular}


Tabla 3. Cuadrados medios y significancia estadística de los valores F evaluados de cinco híbridos de maíz amarillo duro

\begin{tabular}{lrrrrrrrrr}
\hline Factor de & \multicolumn{10}{c}{ Variables } \\
\cline { 2 - 10 } variación & \multicolumn{1}{c}{ FM } & \multicolumn{1}{c}{ FF } & \multicolumn{1}{c}{ AP } & \multicolumn{1}{c}{ AM } & \multicolumn{1}{c}{ DT } & LM & DM & HM & \multicolumn{1}{c}{ PM } \\
\hline A & $11.8^{* *}$ & $15.70^{* *}$ & $12653.08^{* *}$ & $4546.93^{* *}$ & $65.49^{* *}$ & $13575.37^{* *}$ & 1.98 & $35.85^{* *}$ & $18903.48^{* *}$ \\
C.V. (\%) & 1.09 & 1.68 & 6.88 & 11.02 & 9.47 & 10.23 & 6.46 & 9.08 & 21.2 \\
Media & 57.8 & 58.4 & 208.5 & 120.11 & 21.05 & 171.32 & 47.92 & 14.95 & 202.63 \\
\hline
\end{tabular}

$*^{* *}=$ significancia estadística al nivel del 0.05 y 0.01 , respectivamente. $\mathrm{A}=$ híbridos

$\mathrm{CV}=$ coeficiente de variación, $\mathrm{FM}=$ floración masculina; $\mathrm{FF}=$ floración femenina; $\mathrm{AP}=$ altura de planta; AM=altura de mazorca; DT= diámetro de tallo; LM=longitud de mazorca; DM=diámetro de mazorca; $\mathrm{HM}=$ hileras de la mazorca; $\mathrm{PM}=$ peso de mazorca.

El híbrido simple CL02450 x CML287 sobresalió en años anteriores en varios trabajos de investigación, porque posee ventajas agronómicas importantes de arquetipo de planta compacta con orientación de hojas erectas, que permitiría aumentar la densidad de plantas. Por estas bondades, Se afianza como un firme candidato para ser liberado e inscrito en el registro de cultivares comerciales.

La comparación de medias, para las demás variables (floración masculina y femenina, altura de planta y de inserción de mazorca, longitud de mazorca, diámetro de tallo y mazorca, hileras de mazorca y peso de mazorcas), muestran que no existen diferencias significativas entre el híbrido comercial híbrido INIA 619-Megahíbrido y el híbrido CL02450 x CML287 (tabla 4). Esto podría suceder porque al establecer experimentos en diferentes ambientes, el comportamiento de los híbridos en evaluación será diferente, para algunos de ellos, en particular, o en general, en todos los ambientes (Nole, 2012).

Las mejores características obtenidas, con respecto a días a la floración masculina $\mathrm{y}$ femenina, se encontraron en el híbrido AGRHICOL X B-8010. Estos resultados fueron inferiores a los obtenidos por Chura y Tejada
(2014) y se pueden atribuir a la fertilidad de los suelos de la zona de estudio y las características ambientales (Vasco et al., 2017).

Para altura de planta, el híbrido que presentó valor superior fue el INIA 619 Megahíbrido. Una tendencia similar se encontró en Quimi (2015), quien para híbrido comercial AG-003 mostró el máximo valor de altura con 2.49 metros, bajo condiciones de Ecuador. También, Suclupe y Campos (2018) encontraron, para INIA 619 Megahíbrido, una altura de $228.15 \mathrm{~cm}$, en una misma condición de instalación de experimento. Para altura a la mazorca, el híbrido CL02450 x CLM 287 mostró un valor superior a los demás híbridos. Por otro lado, se observó el mayor diámetro de tallo y longitud de mazorca en el genotipo INIA 619 Megahíbrido. Estos resultados fueron inferiores a los reportados por Vasco et al. (2017), quienes encontraron, para híbridos de maíz en condiciones del litoral ecuatoriano, una longitud de $193 \mathrm{~mm}$ y diámetro de tallo de 53 $\mathrm{mm}$. Adicionamlmente, se evideció el mayor diámetro de la mazorca con el híbrido CL12450xCHL287. Finalmente, el mayor número de hilas por mazorca fue representado por el híbrido HML161XCL02410 y el mayor peso de mazorca se reportó con el híbrido INIA 619 Megahíbrido. 
Tabla 4. Comparación de medias de variables agronómicas de cinco híbridos de maíz amarillo duro. Picsi, Lambayeque, Perú. Verano, 2017

\begin{tabular}{|c|c|c|c|c|c|c|c|c|c|}
\hline Híbrido & $\begin{array}{l}\text { FM } \\
\text { (d) }\end{array}$ & $\begin{array}{l}\text { FF } \\
\text { (d) }\end{array}$ & $\begin{array}{c}\mathrm{AP} \\
(\mathrm{cm})\end{array}$ & $\begin{array}{l}\mathrm{AM} \\
(\mathrm{cm})\end{array}$ & $\begin{array}{c}\text { DT } \\
(\mathrm{mm})\end{array}$ & $\begin{array}{c}\text { LM } \\
(\mathrm{mm})\end{array}$ & $\begin{array}{c}\mathrm{DM} \\
(\mathrm{mm})\end{array}$ & HM & $\begin{array}{l}\text { PM } \\
\text { (gr) }\end{array}$ \\
\hline CL02450x CML287 & $59 a$ & $60 \mathrm{ab}$ & $225 \mathrm{a}$ & $128 \mathrm{a}$ & $21 \mathrm{bc}$ & $161 \mathrm{c}$ & $48.2 \mathrm{a}$ & $15 b$ & $205 \mathrm{~b}$ \\
\hline CLRYNO17x CL02450 & $59 \mathrm{a}$ & $58 \mathrm{~b}$ & $203 \mathrm{~b}$ & $124 \mathrm{a}$ & $20 \mathrm{~cd}$ & $162 \mathrm{c}$ & $48.1 \mathrm{a}$ & $15 b$ & $193 \mathrm{bc}$ \\
\hline CML161xCL02410 & $57 \mathrm{~b}$ & $58 \mathrm{~b}$ & $202 \mathrm{~b}$ & $125 \mathrm{a}$ & $20 \mathrm{~d}$ & $156 \mathrm{c}$ & $47.9 \mathrm{a}$ & $16 \mathrm{a}$ & $178 \mathrm{c}$ \\
\hline INIA 619 - Megahíbrido & $60 \mathrm{a}$ & $61 \mathrm{a}$ & $228 \mathrm{a}$ & $122 \mathrm{a}$ & $23 \mathrm{a}$ & $201 \mathrm{a}$ & $47.9 \mathrm{a}$ & $14 \mathrm{bc}$ & $237 \mathrm{a}$ \\
\hline AGRHICOL XB-8010 & $56 \mathrm{~b}$ & $56 \mathrm{c}$ & $185 \mathrm{c}$ & $102 \mathrm{~b}$ & $22 \mathrm{~d}$ & $176 \mathrm{~b}$ & $47.6 \mathrm{a}$ & $14 \mathrm{c}$ & $199 \mathrm{bc}$ \\
\hline D.M.S (0.05) & 1.42 & 2.21 & 8.76 & 8.09 & 1.21 & 10.7 & 1.89 & 0.83 & 26.13 \\
\hline
\end{tabular}

Las medias con la misma letra dentro de cada columna son iguales estadísticamente (Tukey, $\mathrm{P}=0.05$ ).

$\mathrm{FM}=$ floración masculina; $\mathrm{FF}=$ floración femenina; $\mathrm{AP}=$ altura de planta; $\mathrm{AM}=$ altura de mazorca; $\mathrm{DT}=$ diámetro de tallo; $\mathrm{LM}=$ longitud de mazorca; $\mathrm{DM}=$ diámetro de mazorca; $\mathrm{HM}=$ hileras de la mazorca; $\mathrm{PM}=$ =eso de mazorca.

\section{CONCLUSIONES}

El híbrido promisorio CL02450 x CML287, con 9 $704.8 \mathrm{~kg} / \mathrm{ha}$ de grano, tiene productividad superior a los otros cuatro tratamientos $\mathrm{y}$, a su vez, tiene rendimiento similar al híbrido promisorio CLRYNO17 x CL02450, que rindió 8 $938.6 \mathrm{~kg} / \mathrm{ha}$.

Los híbridos simples promisorios muestran un rendimiento superior a los híbridos comerciales de la zona, con un DMS de $1337 \mathrm{Kg}$ ha-1. Por ende, existien diferencias significativas de superioridad, en rendimiento de los híbridos promisorios de maíz amarillo duro CL02450 x CML287 y CLRYN017 x CL02450, con rendimientos de 9704.8 y $8938.6 \mathrm{~kg}$ ha-1, respectivamente.

El híbrido promisorio CL02450 x CML287 posee características productivas y agronómicas para ser un futuro híbrido comercial para la costa norte peruana.

\section{Declaración de intereses}

Ninguna.

\section{Referencias}

Banda, S.E. (2019). Comparativo de rendimiento de 03 híbridos promisorios de maíz amarillo duro (Zea mays L.) y 5 testigos comerciales en el distrito de Batan grande-Santa Clara. Tesis de pregrado, Universidad Nacional Pedro Ruíz Gallo. https://repositorio.unprg.edu.pe/bitstream/handle/2 0.500.12893/8392/BC4795\%20DIAZ\%20BANDA.pdf?sequence=1\&isAllo wed $=\mathrm{y}$

Carballo, C.A. \& Marquez S.P. (1970). Comparación de variedades de Maíz de El Bajio y la meseta central por su rendimiento y estabilidad. Agro-Ciencias, 1, 129146.

Caretas (2021). Perú incrementó su importación de maíz amarillo duro en US\$521 millones en el primer trimestre del año. Recuperado de: https://caretas.pe/economia/peru-incremento-suimportacion-de-maiz-amarillo-duro-en-us-521millones-en-el-primer-trimestre-del-ano/. Leído el 12 de octubre de 2021.

Chura, J. \& Tejada, J. (2014). Comportamiento de híbridos de maíz amarillo duro en la localidad de La Molina, Perú. Idesia (Chile), 32(1), 113-118.

Cordova, H.S. (1978). Uso de parámetros de estabilidad para evaluar el comportamiento de variedades. Guatemala, ICTA 35 p.

Gordon, R., Camargo, I., Franco, J. \& González, A. (2006). Evaluación de la adaptabilidad y estabilidad de 14 
híbridos de maíz, Azuero. Panamá. Agronomía Mesoamericana, 17 (2), 189-199.

Hortus (2020). El maíz es el cultivo más importante en extensión para el Perú. https://www.hortus.com.pe/detalle-noticia/el-maizes-el-cultivo-mas-importante-en-extension-para-elperu. Leido el 12 de octubre de 2021.

La cámara (2021). ¿Cuánto crecieron las importaciones peruanas de trigo y maíz entre enero y abril? Recuperado de: https://lacamara.pe/cuanto-crecieron-lasimportaciones-peruanas-de-trigo-y-maiz-entreenero-y-abril/

MIDAGRI (2021). Maíz Amarillo Duro. Boletín de publicación trimestral № 1-2021. Recuperado de: https://cdn.www.gob.pe/uploads/document/file/1924192/C ommodities\%20Maiz\%20Amarillo\%20Duro\%3A\%20enemar\%202021.pdf. Leido el 12 de octubre de 2021).

MINAGRI (2018). Plan Nacional de Cultivos Campaña Agrícola 2018-2019.DGESEP-DEA. https://agroarequipa.gob.pe/images/AGRICOLA/PL AN\%20NACIONAL\%20DE\%20CULTIVOS\%202018 -2019\%20APROBACION.compressed.pdf

Nole, P. (2012). Evaluación agronómica de ocho híbridos experimentales frente a tres híbridos comerciales de maíz.
Tesis de Grado. Universidad Nacional de Loja. LojaEcuador.

Quimi, D. (2015). Interacción genotipo-ambiente de híbridos triples experimentales de maíz (Zea mays L.) en dos zonas del litoral ecuatoriano. Tesis para optar el título profesional, Universidad técnica estatal de Quevedo, Los Ríos, Ecuador, https://repositorio.uteq.edu.ec/bitstream/43000/20/1/ T-UTEQ-0005.pdf

Suclupe, A.A. \& Campos, N. (2018). Comparativo de rendimiento de 03 híbridos promisorios de maíz amarillo duro (Zea mays L.) y 05 testigos en la parte media y alta del Valle Chancay, región Lambayeque. Tesis de grado, Universidad Nacional Pedro Ruíz Gallo. https://repositorio.unprg.edu.pe/bitstream/handle/2 0.500.12893/8351/Suclupe_Vicente_Astrid_Antonella _y_Campos_Espinoza_Nilson.pdf? sequence $=4 \&$ isAl lowed $=y$

Valsal S.K. (1989). Desarrollo de híbridos no convencionales de maíz. XIII Reunión de Maiceros de la zona Andina Chiclayo, Perú, 1988.

Vasco, A., Saenz, C., Vasco-Mora, S. \& Vasco, D. (2017). Comportamiento agronómico y evaluación económica de híbridos de maíz cristalino duro (Zea mays L.) en tres zonas agroecológicas del Litoral ecuatoriano. Siembra, 4(1), 66-75. 